\title{
The Urgent Need to Understand Covid-19 Associated Coagulopathies and the Significance of Thrombotic Prophylaxis in Critically Ill Patients
}

\author{
Nihaal Singh ${ }^{1}$, Ashish Prakash Anjankar², Shivangi Garima ${ }^{3}$ \\ ${ }^{1}$ Jawaharlal Nehru Medical College, Wardha, Maharashtra, India. ${ }^{2}$ Department of Biochemistry, Jawaharlal \\ Nehru Medical College, Wardha, Maharashtra, India. ${ }^{3}$ Dr. D. Y. Patil Medial College and Hospital, Navi \\ Mumbai, Maharashtra, India.
}

\section{ABSTRACT}

\section{BACKGROUND}

COVID-19 is a novel disease caused due to infection from the respiratory pathogen SARS CoV-2 (Severe Acute Respiratory Syndrome Coronavirus 2). It originated from the city of Wuhan in China in December 2019, and spread to become a pandemic in most of the world. The World Health Organization (WHO) declared COVID-19 to be a 'public health emergency of international concern' on January 30, 2020. Since then there have been countless cases and associated fatalities. In this article we focus on one aspect of the underlying conditions that may prove to be fatal in critically ill patients of COVID-19, namely hypercoagulative states and their associated thrombotic complications. We analyse data-driven studies that outline the manifold increase in instances of COVID-19 related thrombotic events and the patient demography that is most affected by it. There are several reflections and critical enumerations of the specific clinical features that patients with COVID-19 associated coagulopathies present with. We analyse and comment on several anecdotal instances of patients presenting with new-onset symptoms of severe large-vessel ischemic strokes and other coagulopathies. We determine how laboratory findings and specific markers can help identify patients most at risk of thrombotic events. These events may range from deep vein thromboses to fatal cerebrovascular accidents. There is an increased stress on how these coagulation problems may relate to other infectious conditions through presentation with similar markers. We also analyse the radiological investigations that such patients yield and identify peculiarities in them. There is further emphasis on the importance and efficacy of thrombotic prophylaxis and anticoagulant therapy in the management and reduction of fatalities. We also attempt to educate clinicians and intensive care providers regarding better management practices to mitigate fatal outcomes, including both pharmacological and supportive interventions.

\section{KEY WORDS}

COVID-19, Stroke, Hypercoagulative State, Large-Vessel Ischemia, Anticoagulation Therapy, Thrombotic Prophylaxis

\section{Corresponding Author:}

Dr. Ashish Prakash Anjankar,

Department of Biochemistry,

Jawaharlal Nehru Medical College,

Wardha-442107, Maharashtra, India.

E-mail:ashish.anjankar@gmail.com

DOI: $10.14260 /$ jemds/2020/516

How to Cite This Article:

Singh N, Anjankar AP, Garima S. The urgent need to understand covid-19 associated coagulopathies and the significance of thrombotic prophylaxis in critically ill patients. J Evolution Med Dent Sci 2020;9(33):2381-2385, $10.14260 /$ jemds/2020/516

Submission 26-04-2020,

Peer Review 07-07-2020,

Acceptance 14-07-2020,

Published 17-08-2020.

Copyright (c) 2020 JEMDS. This is an open access article distributed under Creative Commons Attribution License [Attribution 4.0 International (CC BY 4.0)] 


\section{BACKGROUND}

In December 2020, doctors reported patients with "pneumonia of an unknown aetiology" in the city of Wuhan, in the Hubei province of China. Researchers later confirmed that the patients were infected with a novel strain of coronavirus that closely resembled the coronaviruses from the SARS and MERS epidemics from 2003 and 2012 respectively. They decided to name it the Novel Coronavirus 2019 or nCoV-2019, and were later modified by the World Health Organization to be referred to as SARS CoV-2 (Severe Acute Respiratory Syndrome Coronavirus-2). ${ }^{1}$ This new strain of coronavirus is allegedly the causative agent for a disease we now refer to as COVID-19 or Novel Coronavirus Pneumonia (NCP). This coronavirus is said to be of zoonotic origin, which simply implies that it shows animal-to-human transmission or "jumping". This further establishes that the place of its origin was the Wuhan Live Seafood market, where the trading of live animals, including exotic mammals, runs rampant and unregulated by health authorities. It is hypothesized that the animal vector could have been a pangolin, a bat or both. As of April 20, 2020, there are a total of 24, 16, 135 cases and $1,65,939$ deaths due to the disease, and $6,32,983$ people have successfully recovered from it.

Like most corona viruses, the SARS CoV-2 is a respiratory pathogen and causes cell damage, fibrosis and other complications in the upper respiratory tract as well as in the lungs. The SARS CoV-2 pathogen is less severe as compared to its close cousins SARS and MERS, but exhibits increased virulence. This increased virulence of the SARS CoV-2 can be attributed to 'Pyroptosis', a novel mechanism of inflammatory cell death. ${ }^{2}$ Corona viruses are non-segmented, positive sense, single strand RNA viruses and are members of the Coronaviridae family, within the order Nidovirales.

Most of them show the presence of 4 major structural proteins, which are, namely

1. Spike Surface Glycoproteins.

2. Small envelope proteins.

3. Matrix Proteins.

4. Nucleocapsid Protein. ${ }^{3}$

The structural protein that is most remarkable, and of particular interest to us, is the 'Spike surface Glycoprotein', which has been reported to be capable of binding to the ACEII (Angiotensin Converting Enzyme - II) receptors by virtue of the 'Receptor Binding Domain.' ${ }^{3}$ This ability to bind to the ACEII receptors is what allows the SARS CoV-2 virus to be pathogenic in human hosts. The ACE-II receptors are present in the human body in most major organ systems, but are more abundant in some than the others.

These organ systems contain the receptors (in the descending order of number of receptors present)-

1. The Respiratory System

2. The Gastrointestinal System

3. The lymphatic fluid and Thymus.

4. The Bone Marrow and Spleen.

5. Liver, Kidney, Brain and so on.

The particular abundance of these receptors in the human respiratory system makes it particularly susceptible to infection by the SARS CoV-2 virus. This leads to several clinical manifestations that may range from mild symptoms like fever, dry cough and fatigue to dyspnoea, chest pain and pneumonia in the severe cases. The symptoms are particularly severe and are more likely to result in fatalities in groups with certain identifiable risk factors. These risk factors are

1. Old Age,

2. Underlying Comorbidities, most commonly Diabetes and Hypertension,

3. Immunocompromised states,

4. Cardiac conditions etc.

Cause of death is mainly attributed to ARDS (Acute Respiratory Distress Syndrome) and Multiple Organ Failure due to hypoxia. However, in post mortem analyses, it is found that most cases show occlusions and micro thrombosis in the small vessels of the lungs. ${ }^{4}$ There is also sufficient evidence to show that anticoagulation prophylaxis is associated with decreased mortality in patients with severe coagulopathy in COVID-19 patients. $^{5}$ In this article, we explore the various instances of thrombotic complications that are associated with the patients of COVID-19. We also aim to elaborate on the younger patient population that presents with ischemic stroke of the large blood vessels. Finally, we shall deal with the significance and role of anticoagulation therapy and thrombotic prophylaxis in reducing the mortality associated with COVID-19.

\section{DISCUSSION}

There is a constantly growing body of clinicians, researchers and intensive care specialists that advocates the role of early thrombotic prophylaxis to mitigate patient loss in case of critically ill COVID-19 cases. There have been reports of both arterial and venous thromboembolisms in patients that return positive test results for presence of the SARS CoV-2 virus in their RTR-PCR (Real Time Polymerase Chain Reaction) tests for nasal and lower respiratory tract swabs. This is usually accompanied by associated risk factors such as

- Inflammation.

- Hypoxia.

- Immobilization.

- Diffuse intravascular coagulopathy.

- Secondary infection.

- Mechanical ventilation.

- Central venous catheter.

This points towards the fact that early introduction of anticoagulation therapy in the course of treatments can significantly help prevent mortality in critical cases of COVID19. In a study on critically ill patients under intensive care who constantly received standard thrombotic prophylaxis throughout their treatment duration, Klok et al. ${ }^{6}$ found that $27 \%$ of the patients out of their total sample size developed venous thromboembolisms, whereas $3.4 \%$ developed arterial thromboembolisms, which put the total number of patients with thrombotic complications at a significant ratio of $31 \%$. Another remarkable feature that arose showed that pulmonary embolism was the most commonly exhibited complication in a staggering $81 \%$ of the total patients with coagulopathy seen in almost all cases. ${ }^{7}$

The study conducted by Wang et al with a significant patient population under consideration showed that the risk 
of death due to venous thromboembolism could be associated with certain identifiable risk factors. These risk factors include Old age, mechanical ventilation, bleed disorders, ICU admission and pneumonia with pneumococcal or influenza infection.

$40 \%$ of the total patients in the study were at increased risk for developing venous thromboembolism and out of these, a ratio of $11 \%$ of these "high risk" patients went on to develop venous thromboembolisms without the administration of any sort of thrombotic prophylaxis. This goes on to show us that there needs to be strict monitoring of patients that arrive for COVID-19 treatment with an increased risk of thrombotic conditions and coagulopathies like arterial/venous thromboembolisms and pulmonary embolisms.

A case of a 57-year-old female reported by Razavi, $\mathrm{A}_{\text {et }} \mathrm{a}^{3}$ presented with initial symptoms of pain, redness and tenderness in one leg. She also complained of a mild cough since the last 3 days. She had no underlying illnesses and had no history of any thrombotic events. CT Angiography came clear for a pulmonary thromboembolism, but she later developed a fever and lymphopenia. Tests also showed dilatation and thrombosis in the paired veins of legs along with the popliteal, superficial and left femoral joint veins. There was a clear consensus about no vascular flow which implied the presence of an acute Deep Vein Thrombosis (DVT). The patient's nasal swab came positive for SARS CoV-2 nucleic acid by the RT-PCR method, and her chest X-ray showed bilateral opacities with patchy ground glass appearance with opacities which are consistent with the COVID-19 associated pneumonia. Her treatment regimen included

- Heparin $80 \mathrm{IU} / \mathrm{kg}$ IV bolus.

- Chloroquine $400 \mathrm{mg}$ once.

- Lopinavir $400 \mathrm{mg}$ twice daily.

- Ritonavir $400 \mathrm{mg}$ twice daily.

On the third day post commencement of treatment with anticoagulants along with antiviral therapy, the patient was free of fever, showed no selling and tenderness and tested negative for the SARS CoV-2 nucleic acid. The patient was healthy without any complications till the date of the publication. ${ }^{8}$

\section{Large-Vessel Stroke and Coagulation Abnormalities as Features of COVID-19}

There have been varied instances of COVID-19 patients presenting with acute large vessel stroke like symptoms and progressing to full-blown ischemic stroke in critical vessels. There is a definitive consensus among experts suggesting that COVID-19 is closely associated with complications of Vascular Endothelial Dysfunction and Coagulopathy. ${ }^{9}$ Thus, it is of utmost importance to acknowledge these findings and familiarize ourselves with the various surface and diagnostic features associated with this patient population.

The occurrence of stroke among patients under hospital admission for COVID-19 was about 5\% and the youngest patient in the sample set was 55 years of age, as suggested by a retrospective data-driven study from the COVID-19 outbreak from Wuhan, China. ${ }^{10}$ In contrast, a total of five patients younger than 50 years of age sought hospital admission with new-onset symptoms of large vessel ischemic stroke, as reported by researchers at a health service facility, and all of them tested positive for COVID-19. This is a significant increase over the 0.73 patients in the same age group that the service treated for large vessel stroke, every two weeks, over the previous 12 months. To confirm the severity of the largevessel stroke, their mean NIHSS (National Institute of Health Stroke Scale) score was found to be 17 . This score is measured on a scale of 0 to 42 , in ascending order of severity. ${ }^{11}$

A case of a 33 year-old woman who presented with cough, headache, and flu-like symptoms was reported by Oxley $\mathrm{T}$ et $\mathrm{al}^{11}$, and over the subsequent time of about 28 hours postpresentation, the patient developed progressive dysarthria, along with numbness and weakness in both her left arm and leg. This occurred over a period of 28 hours. Upon presentation, her NIHSS (National Institute of Health Stroke Scale) score was 19. She tested positive for the SARS CoV-2 nucleotide, based on patchy ground-glass opacities appearing in lung apices bilaterally. Subsequent Computed Tomography (CT) and CT Angiography revealed "partial infarction of the right middle cerebral artery with a partially occlusive thrombus in the right carotid artery at the cervical bifurcation." 11 This is closely reminiscent of the various cases of thrombotic complications that we have previously described in the form of Deep Vein Thromboses, and gives us some insight into how COVID-19 may cause coagulopathies of both in both mild and severe forms.

The above mentioned case was managed with initiation of antiplatelet therapy, and was later replaced by anticoagulation therapy. The source of the thrombus couldn't be determined even after echocardiography, in combination with head and neck MRI (Magnetic Resonance Imaging). On the $10^{\text {th }}$ day postadmission, the thrombus was resolved in its entirely, confirmed by repeat CT Angiography, and the patient was subsequently referred to a rehabilitation facility. ${ }^{11}$

An anecdotal account published by Avula $A$ et al ${ }^{12}$ describes several COVID-19 patient profiles that presented with, or developed stroke like complications. They recount a patient that was intubated due to hypoxic respiratory failure, and a computed tomography (CT) of the head to assess altered mental status depicted acute infarct like features due to graywhite differentiation loss at the left occipital and parietal lobes. Further, "progressive large acute infarct of the left MCA territory with hyperdense appearance of left MCA vessels" was demonstrated with a repeat CT of the head. This distinctly pointed towards acute thrombotic infarct. ${ }^{12}$ This account goes on to cement the hypothesis of hypercoagulative states and their association with COVID-19. It also gives us an insight into how this may affect cerebrovascular functioning. There have been multiple reports of cerebrovascular accidents along with other neurological manifestations in patients with severe infection in recent literature. ${ }^{13}$ The patient in the above study ${ }^{12}$ was determined to be unfit for thrombolysis as well as neurointervention and was thus managed with aspirin and supportive treatment. ${ }^{12}$

\section{Laboratory Findings Supportive of Hypercoagulative States in COVID-19}

To consolidate our knowledge about the diagnostic features of patients that exhibit hypercoagulative states, we shall look at some common trends in laboratory findings of such patients. Typical findings in COVID-19 patients that present with coagulopathies involve, but are not limited to- 
1. Increased D-dimer concentrations. ${ }^{9}$

2. Comparatively mild decrease in platelets. ${ }^{9}$

3. Prolonged prothrombin time. 9

4. Mean fibrinogen concentrations are raised and show sudden decrease shortly before death. ${ }^{14}$

5. Plasma antithrombin concentrations are lower in nonsurvivors, but drop to a lower limit of only $80 \%$ of normal. ${ }^{14}$

6. Rise in Aspartate Aminotransferase (AST) levels.

7. Rise in Alanine Transaminase (ALT) levels.

8. Increase in $\mathrm{C}$ reactive protein levels.

9. Increase in Lactate Dehydrogenase (LDH) levels. ${ }^{9}$

10. Sharp increase in Ferritin Concentrations. ${ }^{9}$

11. Increased concentrations of proinflammatory cytokines. ${ }^{15}$

12. Increased Interleukin concentrations. ${ }^{15}$

COVID-19 and its associated complications closely resemble conditions with systemic coagulopathies, such as severe infections, with disseminated intravascular coagulation (DIC), or thrombotic microangiopathy. ${ }^{16}$ Although several features point towards COVID-19 associated coagulopathy being an amalgamation of regional pulmonary thrombotic microangiopathy and low-grade Disseminated Intravascular Coagulation (DIC) ${ }^{16}$, COVID-19 has several distinct features that sets it apart in terms of a separate condition.

In patient populations with severe COVID-19, cytokine storms have been documented, which exhibit copious release of cytokines and chemokines. These factors may also modulate the coagulation pathways and play a key role in generating coagulative disorders. Raised concentrations of factors such as Proinflammatory Cytokines and Interleukins may induce TFE (Tissue Factor Expression) on mononuclear cells, leading to coagulation initiation and thrombin production. They may also suppress endogenous anticoagulant pathways by mediating these processes. These factors include TNF- $\alpha$ (tumour necrosis factor - alpha), IL-1, and IL-6.15

Thrombocytopenia has been dismissed as a conclusive indicator of disease progression and adverse outcomes in cases of COVID-19.17

\section{Management Strategies of COVID-19 in Accordance with Associated Coagulopathies}

COVID-19 is distinctly and categorically associated with a hypercoagulative state and experts suggest that it must be treated in presence of a high dose thrombotic prophylaxis regimen, even when there is an absence of randomized evidence. It is absolutely imperative, that the patients that are at higher risk for thrombotic events and have had thrombotic complications in the past, be given more attention, due to the fact that they are at a much higher risk for poorer prognosis.

Monitoring of coagulopathy in cases of severe COVID-19 should be done by measuring certain indicators every 2 to 3 days, namely, Prothrombin time, Platelet count, and D-dimer concentrations. ${ }^{17}$

In a study that came out of China, there is an evaluation of the thrombotic parameters, which is summarized by Joey $\mathrm{E}$ (2020), as follows: "They found that if the SIC score was greater than or equal to 4 and the patients had received heparin, there was a decrease in their 28 day mortality from
$64.2 \%$ to $40 \%$. The number needed to treat with all its limitations were just 4.1 If the SIC score wasn't elevated; it really didn't make a difference. The D-dimer also held its own if it was greater than 6 . When this is the case, patients who received heparin had a mortality of $32.8 \%$ versus $54.8 \%$ without it (NNT=5.1)."

Conclusive evidence suggests that use of anticoagulation strategies involving increased prophylactic doses of Low Molecular Weight Heparin (LMWH) are especially successful in management of severely ill COVID-19 patients with Venous Thromboembolism and in reducing mortality.

The progression to later stages of the diseases sees a rise in complications, including renal failure, respiratory failure and liver dysfunction. These complications could adversely affect, hamper or alter the venous thromboembolisms and bleed status of the patients. Thus, these patients must be regularly assessed and their risk for thrombotic events must be constantly evaluated to ensure proper management and recovery. For cases that present with a higher risk for bleeds, anticoagulation therapy must be monitored closely and mechanical compression methods, like compression stockings and intermittent pneumatic compression must be considered as options.

\section{CONCLUSIONS}

There is a compelling body of evidence that suggests that everyone involved as treatment providers for the COVID-19 pandemic must be properly educated regarding the significance of thrombotic prophylaxis and use of anticoagulants for better management of critically ill patients. There have been several reports where it is vividly established that there is an increased risk to patients who are at a higher risk for coagulopathies and associated complications like venous thromboembolisms and pulmonary embolisms. The use of anticoagulation prophylaxis in these high risk cases, as well as in normal cases will ensure the mitigation of a risk for mortality and will also decrease the risk for development of such thrombotic complications. This article also aims at educating the layman about the risks associated with the COVID-19 infection, especially to those who fall into a group of individuals with factors that dictate higher risk to them. Proper precautions and choosing the right strategies in the course of treatment can have a significant impact on the numbers of critically ill patients that proceed to complications that are generally fatal to them. It is also imminent that clinicians and intensive care specialists use their own expertise and rationale to identify patients that are at a higher risk of developing these complications and manage them accordingly in the presence of right prophylactic measures.

Financial or Other Competing Interests: None.

\section{REFERENCES}

[1] Wang T, Chen R, Liu C, et al. Attention should be paid to venous thromboembolism prophylaxis in the management of COVID-19. Lancet Haematol 2020;7(5):e362-3. 
[2] Razavi A, Davoodi L, Taghavi M, et al. COVID-19 presented with deep vein thrombosis: an unusual case report. J Investig Med High Impact Case Rep 2020;8:2324709620931239.

[3] Tang N, Bai H, Chen X, et al. Anticoagulant treatment is associated with decreased mortality in severe coronavirus disease 2019 patients with coagulopathy. J Thromb Haemost 2020;18(5):1094-9.

[4] WHO. Coronavirus. WHO 2020. [online] https://www.who.int/emergencies/diseases/novelcoronavirus-2019.

[5] Joe E. Anticoagulation In COVID-19. 2020. [online] https://www.eddyjoemd.com/2020/04/anticoagulation -in-covid-19.html.

[6] Klok FA, Kruip MJHA, van der Meer NJM, et al. Incidence of thrombotic complications in critically ill ICU patients with COVID-19. Thromb Res 2020;191:145-7.

[7] Yang M. Cell pyroptosis, a potential pathogenic mechanism of 2019-nCoV infection. SSRN Electronic Journal 2020.

[8] Tian S, Xiong Y, Liu H, et al. Pathological study of the 2019 novel coronavirus disease (COVID-19) through postmortem core biopsies. Mod Pathol 2020;33(6):10071014.

[9] Zhou F, Yu T, Du R, et al. Clinical course and risk factors for mortality of adult inpatients with COVID-19 in Wuhan, China: a retrospective cohort study. Lancet 2020;395(10229):1054-62.
[10] Li Y, Wang M, Zhou Y, et al. Acute cerebrovascular disease following COVID-19: a single center, retrospective, observational study. SSRN Electronic Journal 2020.

[11] Oxley T, Mocco J, Majidi S, et al. Large-vessel stroke as a presenting feature of Covid-19 in the young. N Engl J Med 2020;382(20):e60.

[12] Avula A, Nalleballe K, Narula N, et al. COVID-19 presenting as stroke. Brain Behav Immun 2020;87:115-9.

[13] Mao L, Jin H, Wang M, et al. Neurologic manifestations of hospitalized patients with coronavirus disease 2019 in Wuhan, China. JAMA Neurol 2020;77(6):1-9.

[14] Tang N, Li D, Wang X, et al. Abnormal coagulation parameters are associated with poor prognosis in patients with novel coronavirus pneumonia. J Thromb Haemost 2020;18(4):844-7.

[15] Mehta P, McAuley D, Brown M, et al. COVID-19: consider cytokine storm syndromes and immunosuppression. Lancet 2020;395(10229):1033-4.

[16] Levi M, Thachil J, Iba T, et al. Coagulation abnormalities and thrombosis in patients with COVID-19. Lancet Haematol 2020;7(6):e438-40.

[17] Thachil J, Tang N, Gando S, et al. ISTH interim guidance on recognition and management of coagulopathy in COVID19. J Thromb Haemost 2020;18(5):1023-6. 